Real Analysis Exchange

Vol. 22(1), 1996-97, pp. 350-361

Thierry De Pauw*, Département de Mathématique, Université Catholique de Louvain, Chemin du Cyclotron 2, B-1348 Louvain-la-Neuve, Belgium

\title{
A CONCEPT OF GENERALIZED ABSOLUTE CONTINUITY FOR THE $\mathcal{F}$-INTEGRAL
}

\begin{abstract}
We define a concept of generalized absolute continuity for additive functions of figures. This makes it possible to give a descriptive definition of the $\mathcal{F}$-integral introduced in [6]. Finally, we discuss a possible extension to additive functions of sets of bounded variation.
\end{abstract}

Two definitions of multidimensional generalized Riemann integral were introduced in [6] and [5], mainly in order to integrate the divergence of vector fields with singularities. We will call these integrals the $\mathcal{F}$-integral and BVintegral respectively. Their definitions are closely related. In fact, they differ only by the class of domains allowed: for the $\mathcal{F}$-integral this is the class of all figures in $\mathbb{R}^{N}$ (i.e. finite unions of nondegenerate intervals), while the BVintegral integrates functions defined on sets of bounded variation in De Giorgi's sense.

In order to provide descriptive definitions for both integrals, a concept of derivability of continuous additive functions of the domains (shortly: charges) was introduced. Together with a notion of "good behavior on sets of measure zero", the derivability almost everywhere ensures that a charge is an indefinite integral (see [6, Theorem 12.3.4] for the $\mathcal{F}$-integral and [3, Theorem 2.6] for the BV-integral).

The question we address in this paper is that of finding a unifying property of "absolute continuity" equivalent to that of being an indefinite integral. We give an answer in the case of $\mathcal{F}$-integration and discuss the technical difficulties arising from the extension to BV-integration (see [3, Question 2.7] and [1, Question 2.6]).

Mathematical Reviews subject classification: Primary: 26A39

Received by the editors August 8, 1995

* Dedicated to my father 


\section{Preliminaries}

An interval in $\mathbb{R}$ is a set of the type $[a, b]:=\{x \in \mathbb{R}: a \leq x \leq b\}$ where $a, b \in \mathbb{R}$; it is called nondegenerate whenever $a<b$. An interval in $\mathbb{R}^{N}$ is a cartesian product of intervals in $\mathbb{R}: \prod_{i=1}^{N}\left[a_{i}, b_{i}\right]$; it is called nondegenerate whenever each $\left[a_{i}, b_{i}\right]$ is nondegenerate. A figure in $\mathbb{R}^{N}$ is a finite union of nondegenerate intervals. The family of all figures in $\mathbb{R}^{N}$ is denoted $\mathcal{F}\left(\mathbb{R}^{N}\right)$, while if $A \in \mathcal{F}\left(\mathbb{R}^{N}\right)$ we set $\mathcal{F}(A):=\left\{B \in \mathcal{F}\left(\mathbb{R}^{N}\right): B \subset A\right\}$. Two figures $A, B \in \mathcal{F}\left(\mathbb{R}^{N}\right)$ are termed nonoverlapping whenever $\operatorname{int}(A) \cap \operatorname{int}(B)=\emptyset$. Given $A, B \in \mathcal{F}\left(\mathbb{R}^{N}\right), A \cup B \in \mathcal{F}\left(\mathbb{R}^{N}\right)$; we also define

$$
A \odot B:=\operatorname{cl}(\operatorname{int}(A) \cap \operatorname{int}(B)) \text { and } \quad A \ominus B:=\operatorname{cl}(A \backslash B) .
$$

A charge in $A \in \mathcal{F}\left(\mathbb{R}^{N}\right)$ is a function $F: \mathcal{F}(A) \longrightarrow \mathbb{R}$ such that $F(B \cup C)=$ $F(B)+F(C)$ whenever $B$ and $C$ are nonoverlapping subfigures of $A$.

Given $x=\left(x_{1}, \ldots, x_{N}\right) \in \mathbb{R}^{N}$, we put $|x|:=\max \left\{\left|x_{1}\right|, \ldots,\left|x_{N}\right|\right\}$. When $E \subset \mathbb{R}^{N}$, we let $d(E):=\sup \{|x-y|: x, y \in E\}$ and we denote $|E|$ the Lebesgue measure of $E$. We let $\mathcal{H}^{N-1}$ be the $(N-1)$-dimensional Hausdorff measure in $\mathbb{R}^{N}$ and put $\|A\|:=\mathcal{H}^{N-1}(\partial A)$ whenever $A \in \mathcal{F}\left(\mathbb{R}^{N}\right)$. A set is thin if it is the union of countably many sets with finite $\mathcal{H}^{N-1}$ measure.

The regularity of a figure $A \in \mathcal{F}\left(\mathbb{R}^{N}\right), r(A)$, is defined as follows:

$$
r(A):=\left\{\begin{array}{cl}
\frac{|A|}{\|A\| d(A)} & \text { if }\|A\| d(A)>0 \\
0 & \text { otherwise. }
\end{array}\right.
$$

It is related to the usual notion of regularity by the formula $(2 N r(A))^{N} \leq$ $\frac{|A|}{d(A)^{N}}$. Unless specified otherwise, in the rest of this paper $A$ will be a fixed figure in $\mathbb{R}^{N}$. A partition in $A$ is a collection (possibly empty) $\left\{\left(A_{1}, x_{1}\right), \ldots\right.$, $\left.\left(A_{p}, x_{p}\right)\right\}$ where the $A_{i}$ 's are nonoverlapping subfigures of $A$ and $x_{i} \in A_{i}$ for $i \in\{1, \ldots, p\}$. A gage in $A$ is a function $\delta: A \longrightarrow \mathbb{R}_{+}$such that its null set $N_{\delta}:=\left\{x \in \mathbb{R}_{+}: \delta(x)=0\right\}$ is thin. A caliber is a sequence $\left(\eta_{j}\right)_{j \in \mathbb{N}^{*}} \subset \mathbb{R}_{+} \backslash\{0\}$.

Let $\varepsilon>0, \eta \equiv\left(\eta_{j}\right)_{j \in \mathbb{N}^{*}}$ a caliber, $\delta$ a gage in $A$ and $E \subset A$ an arbitrary set. We say that a partition $\left\{\left(A_{1}, x_{1}\right), \ldots,\left(A_{p}, x_{p}\right)\right\}$ in $A$ is:

(i) $\varepsilon$-regular if $r\left(A_{i}\right) \geq \varepsilon$ for all $i \in\{1, \ldots, p\}$;

(ii) $\delta$-fine if $d\left(A_{i}\right) \leq \delta\left(x_{i}\right)$ for all $i \in\{1, \ldots, p\}$;

(iii) ( $\varepsilon, \eta)$-approximating if $A \ominus \cup_{i=1}^{p} A_{i}$ is the union of finitely many nonoverlapping figures $B_{1}, \ldots, B_{k}$ with $\left\|B_{j}\right\| \leq \frac{1}{\varepsilon}$ and $\left|B_{j}\right| \leq \eta_{j}$ for all $j \in$ $\{1, \ldots, k\}$

(iv) E-tagged if $x_{i} \in E$ for all $i \in\{1, \ldots, p\}$. 
Let $f: A \longrightarrow \mathbb{R}$. We say that $f$ is $\mathcal{F}$-integrable in $A$ if there is a real number $I$ (called its $\mathcal{F}$-integral and also denoted $\int_{A} f$ ) with the following property: given $\varepsilon>0$, one can find a gage $\delta$ in $A$ and a caliber $\eta$ such that

$$
\left|\sum_{i=1}^{p} f\left(x_{i}\right)\right| A_{i}|-I| \leq \varepsilon
$$

for every $\delta$-fine, $\varepsilon$-regular, $(\varepsilon, \eta)$-approximating partition $\left\{\left(A_{1}, x_{1}\right), \ldots\right.$, $\left.\left(A_{p}, x_{p}\right)\right\}$ in $A$. If $f$ is $\mathcal{F}$-integrable in $A$ and $B \in \mathcal{F}(A)$, then $f$ is also $\mathcal{F}$-integrable in $B$; moreover its indefinite integral $F$, defined by $F(B):=\int_{B} f$ whenever $B \in \mathcal{F}(A)$, is a charge.

Let $F: \mathcal{F}(A) \longrightarrow \mathbb{R}$ be an arbitrary charge and $x \in A$. We define the lower derivative (resp. upper derivative) of $F$ at $x$, denoted $F_{\star}(x)\left(\right.$ resp. $\left.F^{\star}(x)\right)$ as follows:

$$
\begin{aligned}
F_{\star}(x) & :=\inf _{\eta>0} \sup _{\delta>0} \inf \left\{\frac{F(X)}{|X|}: X \in \mathcal{F}(A), x \in X, d(X) \leq \delta, r(X) \geq \eta\right\}, \\
F^{\star}(x) & :=\sup _{\eta>0} \inf _{\delta>0} \sup \left\{\frac{F(X)}{|X|}: X \in \mathcal{F}(A), x \in X, d(X) \leq \delta, r(X) \geq \eta\right\} .
\end{aligned}
$$

These are extended real numbers. It is easily seen that $F_{\star}$ and $F^{\star}$ are measurable functions and that $F_{\star}(x) \leq F^{\star}(x)$ (in $[-\infty,+\infty]$ ) whenever $x \in \operatorname{int}(A)$. We say that $F$ is derivable at $x$ if $F_{\star}(x)=F^{\star}(x) \in \mathbb{R}$ and in this case, the common value of the derivatives is denoted $F^{\prime}(x)$.

A charge $F: \mathcal{F}(A) \longrightarrow \mathbb{R}$ is called continuous in $A$ if for every $\varepsilon>0$ there exists $\eta>0$ such that $|F(B)| \leq \varepsilon$ whenever $B \in \mathcal{F}(A)$ with $|B| \leq \eta$ and $\|B\| \leq \frac{1}{\varepsilon}$.

A charge $F: \mathcal{F}(A) \longrightarrow \mathbb{R}$ is called $\mathcal{F} A C_{*}$ in $A$ if it is continuous in $A$ and, given $E \subset A$ with $|E|=0$ and $\varepsilon>0$, there is a gage $\delta$ in $A$ such that $\sum_{i=1}^{p}\left|F\left(A_{i}\right)\right| \leq \varepsilon$ for each $\varepsilon$-regular, $\delta$-fine, $E$-tagged partition $\left\{\left(A_{1}, x_{1}\right), \ldots,\left(A_{p}, x_{p}\right)\right\}$ in $A$.

The following result can be found in [6, Theorem 12.3.4]:

Let $F: \mathcal{F}(A) \longrightarrow \mathbb{R}$ be a charge. $F$ is the indefinite integral of some $\mathcal{F}$ integrable function if and only if it is $\mathcal{F} A C_{*}$ in $A$ and derivable almost everywhere in $A$. In this case, $F(B)=\int_{B} F^{\prime}$ for each $B \in \mathcal{F}(A)$.

We point out the fact that this Theorem implies that each $\mathcal{F}$-integrable function is measurable.

\section{Generalized Absolute Continuity}

We will define a concept of generalized absolute continuity which differs slightly from that of [1] but resembles the one introduced in [2]. 
Definition 2.1 Let $F: \mathcal{F}(A) \longrightarrow \mathbb{R}$ be a charge and $X \subset A$. We say that $F$ is $\mathcal{F}-A C^{\nabla}(X)$ if it enjoys the following property: given $\varepsilon>0$, we can find a gage $\delta$ in $A$ and a positive real number $\eta>0$ such that for every $\delta$-fine, $\varepsilon$-regular, $X$-tagged partitions in $A\left\{\left(A_{1}, x_{1}\right), \ldots,\left(A_{p}, x_{p}\right)\right\}$ and $\left\{\left(B_{1}, y_{1}\right), \ldots,\left(B_{q}, y_{q}\right)\right\}$, if

$$
\left|\left(\cup_{i=1}^{p} A_{i}\right) \triangle\left(\cup_{j=1}^{q} B_{j}\right)\right| \leq \eta
$$

then

$$
\left|\sum_{i=1}^{p} F\left(A_{i}\right)-\sum_{j=1}^{q} F\left(B_{j}\right)\right| \leq \varepsilon .
$$

Definition 2.2 Let $F: \mathcal{F}(A) \longrightarrow \mathbb{R}$ be a charge. By saying that $F$ is $\mathcal{F}-$ $A C G^{\nabla}(A)$, we mean that there is a sequence $\left(X_{n}\right)_{n \in \mathbb{N}}$ of measurable subsets of $A$ such that $A=\cup_{n \in \mathbb{N}} X_{n}$ and $F$ is $\mathcal{F}-A C^{\nabla}\left(X_{n}\right)$ for every $n \in \mathbb{N}$.

From definition 2.1 we infer that $F$ is $\mathcal{F}-A C^{\nabla}(Y)$ whenever it is $\mathcal{F}-$ $A C^{\nabla}(X)$ and $Y \subset X$. In particular, it is not a restriction to assume that the sets $X_{n}$ arising from definition 2.2 are pairwise disjoint.

The following lemma is just a reformulation of [2, Theorem 2.9].

Lemma 2.1 Let $f: A \longrightarrow \mathbb{R}$ be $\mathcal{F}$-integrable in $A$ and $F$ its indefinite integral. Then $F$ is $\mathcal{F}-A C G^{\nabla}(A)$.

Proof. Since $f$ is measurable, the sets

$$
X_{n}:=\{x \in A:|f(x)| \leq n\}
$$

are measurable. Fix $n \in \mathbb{N}$ : we need to show that $F$ is $\mathcal{F}-A C^{\nabla}\left(X_{n}\right)$. We consider the function $\tilde{f}: A \longrightarrow \mathbb{R}$ defined as follows:

$$
\tilde{f}(x):=\left\{\begin{array}{cl}
f(x) & \text { if } x \in X_{n} \\
0 & \text { otherwise }
\end{array}\right.
$$

Being Lebesgue-integrable in $A, \tilde{f}$ is $\mathcal{F}$-integrable in $A$ ([5, Proposition 3.1]). We denote $\tilde{F}$ its indefinite integral. Let $\varepsilon>0$, using Henstock's lemma ([6, Theorem 12.3.2]) we can find a gage $\delta$ in $A$ such that

$$
\sum_{i=1}^{p}\left|f\left(x_{i}\right)\right| A_{i}\left|-F\left(A_{i}\right)\right| \leq \frac{\varepsilon}{8}
$$

and

$$
\sum_{i=1}^{p}\left|\tilde{f}\left(x_{i}\right)\right| A_{i}\left|-\tilde{F}\left(A_{i}\right)\right| \leq \frac{\varepsilon}{8}
$$


for each $\delta$-fine, $\frac{\varepsilon}{8}$-regular partition in $A\left\{\left(A_{1}, x_{1}\right), \ldots,\left(A_{p}, x_{p}\right)\right\}$. Since $\tilde{F}$ is absolutely continuous with respect to Lebesgue's measure, there is $\eta>0$ such that $|\tilde{F}(E)| \leq \frac{\varepsilon}{2}$ for every measurable set $E \subset A$ with $|E| \leq \eta$. Now assume that $\left\{\left(A_{1}, x_{1}\right), \ldots,\left(A_{p}, x_{p}\right)\right\}$ and $\left\{\left(B_{1}, y_{1}\right), \ldots,\left(B_{q}, y_{q}\right)\right\}$ are $\delta$-fine, $\varepsilon$-regular, $X_{n}$-tagged partitions in $A$ (being $X_{n}$-tagged implies that $f\left(x_{i}\right)=\tilde{f}\left(x_{i}\right)$ and $f\left(y_{j}\right)=\tilde{f}\left(y_{j}\right)$ for all $i$ and $\left.j\right)$ and that $\left|\left(\cup_{i=1}^{p} A_{i}\right) \triangle\left(\cup_{j=1}^{q} B_{j}\right)\right| \leq \eta$ :

$$
\begin{aligned}
\mid \sum_{i=1}^{p} F\left(A_{i}\right) & -\sum_{j=1}^{q} F\left(B_{j}\right)\left|\leq \sum_{i=1}^{p}\right| F\left(A_{i}\right)-f\left(x_{i}\right)\left|A_{i}\right|\left|+\sum_{i=1}^{p}\right| \tilde{f}\left(x_{i}\right)\left|A_{i}\right|-\tilde{F}\left(A_{i}\right) \mid \\
& +\left|\tilde{F}\left(\left(\cup_{i=1}^{p} A_{i}\right) \triangle\left(\cup_{j=1}^{q} B_{j}\right)\right)\right| \sum_{j=1}^{q}\left|\tilde{F}\left(B_{j}\right)-\tilde{f}\left(y_{j}\right)\right| B_{j}|| \\
& +\sum_{j=1}^{q}\left|f\left(y_{j}\right)\right| B_{j}\left|-F\left(B_{j}\right)\right| \leq \frac{\varepsilon}{8}+\frac{\varepsilon}{8}+\frac{\varepsilon}{2}+\frac{\varepsilon}{8}+\frac{\varepsilon}{8} \leq \varepsilon
\end{aligned}
$$

The aim of the three following results is to prove that a charge $F$ is derivable almost everywhere in $X$ provided it is $\mathcal{F}-A C^{\nabla}(X)$.

Lemma 2.2 Let $C \subset \operatorname{int}(A)$ be compact, $0<\varepsilon \leq \frac{1}{2 N}$ and $\gamma: C \longrightarrow \mathbb{R}_{+} \backslash\{0\}$. There is an $\varepsilon$-regular, $\gamma$-fine, $C$-tagged partition in $A\left\{\left(C_{1}, x_{1}\right), \ldots,\left(C_{m}, x_{m}\right)\right\}$ such that $C \subset \cup_{i=1}^{m} C_{i}$.

Proof. Let $P$ be any dyadic cube containing $C$. We define a positive function $\delta: P \longrightarrow \mathbb{R}_{+} \backslash\{0\}$ as follows:

$$
\delta(x):= \begin{cases}\min \left\{\gamma(x), \frac{1}{2} \operatorname{dist}(x, \partial A)\right\} & \text { if } x \in C \\ \frac{1}{2} \operatorname{dist}(x, C) & \text { if } x \notin C .\end{cases}
$$

Let $\left\{\left(C_{1}, x_{1}\right), \ldots,\left(C_{l}, x_{l}\right)\right\}$ be any $\delta$-fine partition of $A$ such that the $C_{i}$ 's are dyadic cubes. It is easily seen that the family $\left\{\left(C_{i}, x_{i}\right): x_{i} \in C\right\}$ satisfies the required conditions.

Proposition 2.1 Let $F: \mathcal{F}(A) \longrightarrow \mathbb{R}$ be a charge, $E \subset \operatorname{int}(A)$ a measurable set and assume that $F$ is $\mathcal{F}-A C^{\nabla}(E)$. Given $0<\varepsilon \leq \frac{1}{2 N}, \theta>0, \alpha \in \mathbb{R}$, $\beta \in \mathbb{R}$ and a gage $\delta$ in $A$, one can find an $\varepsilon$-regular, $\delta$-fine, E-tagged partition in $A\left\{\left(C_{1}, x_{1}\right), \ldots,\left(C_{m}, x_{m}\right)\right\}$ such that $\left|\cup_{i=1}^{m} C_{i}\right| \leq|E|+\theta$ and satisfying the two following conditions:

(1) if $E \subset\left\{x \in A: F^{\star}(x) \geq \beta\right\}$ then $F\left(\cup_{i=1}^{m} C_{i}\right)+\varepsilon \geq \beta|E|$; 
(2) if $E \subset\left\{x \in A: F_{\star}(x) \leq \alpha\right\}$ then $F\left(\cup_{i=1}^{m} C_{i}\right)-\varepsilon \leq \alpha|E|$.

Proof. First, we use the continuity of the map

$$
\mathbb{R} \times \mathbb{R} \longrightarrow \mathbb{R}:(x, y) \longmapsto x \cdot y
$$

at the points $(\alpha,|E|)$ and $(\beta,|E|)$ to find some $\eta_{1}>0$ sufficiently small for the following to be true: if $\max \{|x-\alpha|,|y-| E||\} \leq \eta_{1}$ then

$$
|x y-\alpha| E|| \leq \frac{\varepsilon}{2},
$$

and if $\max \{|x-\beta|,|y-| E||\} \leq \eta_{1}$ then

$$
|x y-\beta| E|| \leq \frac{\varepsilon}{2} .
$$

To simplify the notations, put $A_{\beta}^{\star}:=\left\{x \in A: F^{\star}(x) \geq \beta\right\}$ and $A_{\star}^{\alpha}:=\{x \in$ $\left.A: F_{\star}(x) \leq \alpha\right\}$.

If $E \subset A_{\beta}^{\star}$ then to each $j \in \mathbb{N}^{*}$ we associate a set

$$
\begin{gathered}
E_{j}^{\star}:=\{x \in E:(\forall \delta>0)(\exists X \in \mathcal{F}(A)) \text { such that } x \in X, d(X) \leq \delta, \\
\left.r(X) \geq \frac{1}{j} \text { and } F(X) \geq\left(\beta-\eta_{1}\right)|X|\right\} .
\end{gathered}
$$

It is easily observed that

$$
E_{j}^{\star}=E \cap\left(\cap_{k \in \mathbb{N}^{*}} E_{j, k}^{\star}\right)
$$

where we have put

$$
E_{j, k}^{\star}:=\bigcup\left\{X \in \mathcal{F}(A): d(X) \leq \frac{1}{k}, r(X) \geq \frac{1}{j} \text { and } F(X) \geq\left(\beta-\eta_{1}\right)|X|\right\}
$$

for $k \in \mathbb{N}^{*}$. Since the $E_{j, k}^{\star}$ 's are measurable (see [7, Ch. IV, Lemma 4.1]), so are also the $E_{j}^{\star}$ 's. Moreover, the relation $E \subset A_{\beta}^{\star}$ implies that $\cup_{j \in \mathbb{N}^{*}} E_{j}^{\star}=E$.

If $E \not \subset A_{\beta}^{\star}$, we let $E_{j}^{\star}:=E$ for all $j \in \mathbb{N}^{*}$.

If $E \subset A_{\star}^{\alpha}$ then to each $j \in \mathbb{N}^{*}$ we associate a set

$$
\begin{gathered}
E_{\star}^{j}:=\{x \in E:(\forall \delta>0)(\exists X \in \mathcal{F}(A)) \text { such that } x \in X, d(X) \leq \delta, \\
\left.r(X) \geq \frac{1}{j} \text { and } F(X) \leq\left(\alpha+\eta_{1}\right)|X|\right\} .
\end{gathered}
$$

We also infer that the $E_{\star}^{j}$ 's are measurable and that $\cup_{j \in \mathbb{N}^{*}} E_{\star}^{j}=E$. 
If $E \not \subset A_{\star}^{\alpha}$, we let $E_{\star}^{j}:=E$ for all $j \in \mathbb{N}^{*}$.

Now, put $E_{j}:=E_{j}^{\star} \cap E_{\star}^{j}$. This defines an increasing sequence of measurable sets such that $\cup_{j \in \mathbb{N}^{*}} E_{j}=E$. Hence there is a $j_{0} \in \mathbb{N}^{*}$ with the property

$$
\left|E \backslash E_{j_{0}}\right| \leq \frac{\eta_{1}}{3}
$$

Let $\eta_{2}:=\min \left\{\frac{\varepsilon}{2}, \frac{1}{j_{0}}\right\}$. Since $F$ is $\mathcal{F}-A C^{\nabla}(E)$, there is a gage $\delta_{0}$ in $A$ and a $\eta_{3}>0$ such that for every $\delta_{0}$-fine, $\eta_{2}$-regular, $E$-tagged partitions in $A$ $\left\{\left(A_{1}, x_{1}\right), \ldots,\left(A_{p}, x_{p}\right)\right\}$ and $\left\{\left(B_{1}, y_{1}\right), \ldots,\left(B_{q}, y_{q}\right)\right\}$, the relation

$$
\left|\left(\cup_{i=1}^{p} A_{i}\right) \triangle\left(\cup_{j=1}^{q} B_{j}\right)\right| \leq \eta_{3}
$$

implies

$$
\left|\sum_{i=1}^{p} F\left(A_{i}\right)-\sum_{j=1}^{q} F\left(B_{j}\right)\right| \leq \eta_{2} .
$$

Since $E_{j_{0}}$ is measurable, there is an open set $V$ and a closed set $C$ with the following properties:

$$
\begin{gathered}
C \subset E_{j_{0}} \backslash\left(N_{\delta_{0}} \cup N_{\delta}\right) \text { and }\left|E_{j_{0}} \backslash C\right| \leq \frac{\eta_{3}}{4}, \\
E_{j_{0}} \subset V \text { and }\left|V \backslash E_{j_{0}}\right| \leq \min \left\{\theta, \frac{\eta_{1}}{3}, \frac{\eta_{3}}{4}\right\},
\end{gathered}
$$

(recall that $\left|N_{\delta_{0}} \cup N_{\delta}\right|=0$ ).

We can now define a function $\gamma: C \longrightarrow \mathbb{R}_{+} \backslash\{0\}$ by means of the following formula:

$$
\gamma(x):=\min \left\{\delta(x), \delta_{0}(x), \frac{1}{2} \operatorname{dist}(x, \partial V)\right\} .
$$

Apply Lemma 2.2 to $C, \gamma$ and $\varepsilon$ to find a partition in $A\left\{\left(C_{1}, x_{1}\right), \ldots\right.$, $\left.\left(C_{m}, x_{m}\right)\right\} \varepsilon$-regular (also $\eta_{2}$-regular since $\left.\eta_{2} \leq \frac{\varepsilon}{2}\right), \gamma$-fine (also $\delta$ - and $\delta_{0}$-fine), $C$-tagged (also $E$-tagged); moreover,

$$
C \subset \cup_{i=1}^{m} C_{i} \subset V
$$

We claim that this partition enjoys all the required properties. First, from (4) and (3) we deduce that

$$
\left|\cup_{i=1}^{m} C_{i}\right| \leq|V|=\left|E_{j_{0}}\right|+\left|V \backslash E_{j_{0}}\right| \leq|E|+\theta .
$$


We now want to show that $\left\{\left(C_{1}, x_{1}\right), \ldots,\left(C_{m}, x_{m}\right)\right\}$ satisfies condition (1) of the thesis. We consider the family

$$
\begin{aligned}
\mathcal{C}:=\{(X, x): & X \in \mathcal{F}(A), x \in E_{j_{0}}^{\star}, x \in X, r(X) \geq \frac{1}{j_{0}} \\
& \left.d(X) \leq \min \left\{\delta_{0}(x), \frac{1}{2} \operatorname{dist}(x, \partial V)\right\}, F(X) \geq\left(\beta-\eta_{1}\right)|X|\right\} .
\end{aligned}
$$

Since we assume $E \subset A_{\beta}^{\star}$, it is clear that $\{X:(X, x) \in \mathcal{C}$ for some $x\}$ is a Vitali cover of $E_{j_{0}}$ in the sense of [7, Ch. IV '3] and consequently there is a countable subfamily $\left\{\left(X_{i}, x_{i}\right): i \in \mathbb{N}^{*}\right\} \subset \mathcal{C}$ such that the $X_{i}$ 's are pairwise disjoint and

$$
\left|E_{j_{0}} \backslash \cup_{i \in \mathbb{N}^{*}} X_{i}\right|=0
$$

We choose an index $k \in \mathbb{N}^{*}$ sufficiently large for

$$
\left|\left(\cup_{i \in \mathbb{N}^{*}} X_{i}\right) \backslash\left(\cup_{i=1}^{k} X_{i}\right)\right| \leq \min \left\{\frac{\eta_{1}}{3}, \frac{\eta_{3}}{4}\right\} .
$$

We observe that $\left\{\left(X_{1}, x_{1}\right), \ldots,\left(X_{k}, x_{k}\right)\right\}$ is a $\delta_{0}$-fine, $\eta_{4}$-regular (because $\eta_{4} \leq$ $\left.\frac{1}{j_{0}}\right), E$-tagged partition in $A$ and that

$$
\cup_{i=1}^{k} X_{i} \subset \cup_{i \in \mathbb{N}^{*}} X_{i} \subset V
$$

On the other hand, relations (4), (5), (6) and (3) imply that

$$
\begin{aligned}
\mid\left(\cup_{i=1}^{m} C_{i}\right) & \backslash\left(\cup_{i=1}^{k} X_{i}\right)|\leq| V \backslash\left(\cup_{i=1}^{k} X_{i}\right) \mid \\
& \leq\left|E_{j_{0}} \backslash\left(\cup_{i=1}^{k} X_{i}\right)\right|+\left|V \backslash E_{j_{0}}\right| \\
& \leq\left|E_{j_{0}} \backslash\left(\cup_{i \in \mathbb{N}^{*}} X_{i}\right)\right|+\left|\left(\cup_{i \in \mathbb{N}^{*}} X_{i}\right) \backslash\left(\cup_{i=1}^{k} X_{i}\right)\right|+\left|V \backslash E_{j_{0}}\right| \leq \frac{\eta_{3}}{2},
\end{aligned}
$$

and relations (7), (4), (2) and (3) imply that

$$
\left|\left(\cup_{i=1}^{k} X_{i}\right) \backslash\left(\cup_{i=1}^{m} C_{i}\right)\right| \leq|V \backslash C|=\left|V \backslash E_{j_{0}}\right|+\left|E_{j_{0}} \backslash C\right| \leq \frac{\eta_{3}}{2} .
$$

Put these estimations together to obtain

$$
\left|\left(\cup_{i=1}^{m} C_{i}\right) \triangle\left(\cup_{i=1}^{k} X_{i}\right)\right| \leq \eta_{3} .
$$

Hence, from the choice of $\eta_{3}$ and $\delta_{0}$ we infer that

$$
\left|\sum_{i=1}^{k} F\left(X_{i}\right)-\sum_{i=1}^{m} F\left(C_{i}\right)\right| \leq \frac{\varepsilon}{2} .
$$


Furthermore, from the properties of the $X_{i}$ 's we deduce that

$$
F\left(\cup_{i=1}^{m} C_{i}\right)+\frac{\varepsilon}{2} \geq \sum_{i=1}^{k} F\left(X_{i}\right) \geq\left(\beta-\eta_{1}\right)\left|\cup_{i=1}^{k} X_{i}\right|
$$

Next, we want to show that

$$
|| E|-| \cup_{i=1}^{k} X_{i}|| \leq \eta_{1}
$$

First check that

$$
\left|E_{j_{0}}\right|-\left|\cup_{i \in \mathbb{N}^{*}} X_{i}\right| \leq\left|E_{j_{0}} \backslash\left(\cup_{i \in \mathbb{N}^{*}} X_{i}\right)\right|=0,
$$

next, using (7) and (3),

$$
\left|\cup_{i \in \mathbb{N}^{*}} X_{i}\right|-\left|E_{j_{0}}\right| \leq|V|-\left|E_{j_{0}}\right| \leq \frac{\eta_{1}}{3}
$$

so that

$$
|| E_{j_{0}}|-| \cup_{i \in \mathbb{N}^{*}} X_{i} \mid \leq \frac{\eta_{1}}{3} .
$$

Then we obtain (9) in the following way, using (1),(10) and (6):

$$
\begin{aligned}
|| E|-| \cup_{i=1}^{k} X_{i}|| & \leq|| E|-| E_{j_{0}}||+|| E_{j_{0}}|-| \cup_{i \in \mathbb{N}^{*}} X_{i}|| \\
& +|| \cup_{i \in \mathbb{N}^{*}} X_{i}|-| \cup_{i=1}^{k} X_{i}|| \leq \eta_{1} .
\end{aligned}
$$

From (9) and the choice of $\eta_{1}$ we get

$$
\left(\beta-\eta_{1}\right)\left|\cup_{i=1}^{k} X_{i}\right| \geq \beta|E|-\frac{\varepsilon}{2}
$$

which, together with (8), implies that

$$
F\left(\cup_{i=1}^{m} C_{i}\right)+\varepsilon \geq \beta|E| .
$$

This completes the proof of the first part of Proposition 2.1.

In order to prove that $\left\{\left(C_{1}, x_{1}\right), \ldots,\left(C_{m}, x_{m}\right)\right\}$ satisfies condition (2) of the thesis, we should construct another partition $\left\{\left(Y_{1}, y_{1}\right), \ldots,\left(Y_{l}, y_{l}\right)\right\}$ with, among many other ones, the property that $F\left(Y_{j}\right) \leq\left(\alpha+\eta_{1}\right)\left|Y_{j}\right|$. Since the construction is analogous to the first one, we omit the details.

Lemma 2.3 Let $E \subset \mathbb{R}^{N}$ be a measurable set and $\alpha>0$. If $|E|>0$, then there exists a measurable set $\tilde{E} \subset E$ such that $0<|\tilde{E}| \leq \alpha$. 
Proof. We may assume $E$ is bounded. Given $t \in \mathbb{R}$, define $T_{t}:=\left\{x \in \mathbb{R}^{N}\right.$ : $\left.x_{1}<t\right\}$. Observe that the map $\varphi(t):=\left|E \cap T_{t}\right|$ is lipschitz-continuous and $\lim _{t \rightarrow \infty} \varphi(t)=|E|>0, \lim _{t \rightarrow-\infty} \varphi(t)=0$. Hence, there is some $t_{0} \in \mathbb{R}$ such that $\varphi\left(t_{0}\right)=\min \{\alpha,|E|\}$. The set $\tilde{E}:=E \cap T_{t_{0}}$ satisfies the thesis.

Proposition 2.2 Let $F: \mathcal{F}(A) \longrightarrow \mathbb{R}$ be a charge, $X \subset A$ a measurable set and assume that $F$ is $\mathcal{F}-A C^{\nabla}(X)$. Then $F$ is derivable at almost every point of $X$.

Proof. Let $E$ be the set of points in $X \cap \operatorname{int}(A)$ where $F$ is not derivable. We define

$$
\begin{aligned}
E_{-} & :=\left\{x \in X \cap \operatorname{int}(A): F_{\star}(x)=-\infty\right\} \\
E^{+} & :=\left\{x \in X \cap \operatorname{int}(A): F^{\star}(x)=+\infty\right\} \\
E_{\alpha, \beta} & :=\left\{x \in X \cap \operatorname{int}(A): F_{\star}(x) \leq \alpha<\beta \leq F^{\star}(x)\right\}
\end{aligned}
$$

so that

$$
E=E_{-} \cup E^{+} \cup\left(\cup_{\alpha, \beta \in \mathbb{Q}} E_{\alpha, \beta}\right) .
$$

First, we want to prove that $\left|E_{-}\right|=0$. In order to get a contradiction, suppose $\left|E_{-}\right|>0$. Using the fact that $F$ is $\mathcal{F}-A C^{\nabla}(X)$, choose any gage $\delta$ in $A$ and $\eta>0$ in order that for each $\frac{1}{4 N}$-regular, $\delta$-fine partitions in $A$ $\left\{\left(A_{1}, x_{1}\right), \ldots,\left(A_{p}, x_{p}\right)\right\}$ and $\left\{\left(B_{1}, y_{1}\right), \ldots,\left(B_{q}, y_{q}\right)\right\}$, if $\left|\left(\cup_{i=1}^{p} A_{i}\right) \triangle\left(\cup_{j=1}^{q} B_{j}\right)\right|$ $\leq \eta$ then $\left|\sum_{i=1}^{p} F\left(A_{i}\right)-\sum_{j=1}^{q} F\left(B_{j}\right)\right| \leq \frac{1}{4 N}$. Apply Lemma 2.3 to $E_{-}$and $\alpha=\frac{\eta}{2}$ to find a measurable set $\tilde{E}_{-} \subset E_{-}$such that $0<\left|\tilde{E}_{-}\right| \leq \frac{\eta}{2}$. Next apply Proposition 2.1 to $F, \varepsilon=\frac{1}{4 N}, \theta=\left|\tilde{E}_{-}\right|, \alpha=-\frac{1}{N|\tilde{E}|}$ and the gage $\delta$ we kept from the fact that $F$ is $\mathcal{F}-A C^{\nabla}(X)$. We get a $\frac{1}{4 N}$-regular, $\delta$-fine, $X$-tagged partition in $A\left\{\left(C_{1}, x_{1}\right), \ldots,\left(C_{m}, x_{m}\right)\right\}$ such that $\left|\cup_{i=1}^{m} C_{i}\right| \leq 2\left|\tilde{E}_{-}\right| \leq \eta$ and hence, from the choice of $\delta$ and $\eta$,

$$
\left|F\left(\cup_{i=1}^{m} C_{i}\right)\right| \leq \frac{1}{4 N}
$$

On the other hand, from Proposition 2.1 (2), this partition satisfies also

$$
F\left(\cup_{i=1}^{m} C_{i}\right) \leq \alpha\left|\tilde{E}_{-}\right|+\varepsilon=-\frac{3}{4 N} .
$$

This is in contradiction with (11). The proof that $\left|E^{+}\right|=0$ is very similar and we omit the details.

Finally, fix $\alpha, \beta \in \mathbb{Q}$ such that $\alpha<\beta$. We need to prove that $\left|E_{\alpha, \beta}\right|=0$. Given any $\frac{1}{4 N} \geq \varepsilon>0$, we find through Proposition 2.1 a figure $\cup_{i=1}^{m} C_{i}$ in $A$ 
such that

$$
F\left(\cup_{i=1}^{m} C_{i}\right)-\varepsilon \leq \alpha\left|E_{\alpha, \beta}\right| \leq \beta\left|E_{\alpha, \beta}\right| \leq F\left(\cup_{i=1}^{m} C_{i}\right)+\varepsilon .
$$

Hence,

$$
|\alpha-\beta| \cdot\left|E_{\alpha, \beta}\right| \leq 2 \varepsilon
$$

since $\varepsilon>0$ is arbitrary small and $\alpha \neq \beta$, we get $\left|E_{\alpha, \beta}\right|=0$.

The following is just a reformulation of [1, Lemma 2.2]. We omit the proof.

Lemma 2.4 Let $F: \mathcal{F}(A) \longrightarrow \mathbb{R}$ be a continuous charge. If $F$ is $\mathcal{F}-$ $A C G^{\nabla}(A)$, then $F$ is $\mathcal{F} A C^{\star}$ in $A$.

Theorem 2.1 Let $F: \mathcal{F}(A) \longrightarrow \mathbb{R}$ be a charge. The following conditions are equivalent:

(1) $F$ is continuous and $\mathcal{F}-A C G^{\nabla}(A)$;

(2) $F$ is $\mathcal{F} A C^{\star}$ in $A$ and almost everywhere derivable in $A$;

(3) $F$ is the indefinite integral of some $\mathcal{F}$-integrable function in $A$.

Proof. (1) implies (2) in view of Proposition 2.2 and Lemma 2.4. (2) was already known to be equivalent to (3). Finally, (3) implies (1) from Lemma 2.1.

Question 2.1 Here we define generalized absolute continuity as in [1]. Let $F: \mathcal{F}(A) \longrightarrow \mathbb{R}$ be a charge and $X \subset A$. We say that $F$ is $\mathcal{F}-A C^{\star}(X)$ if given $\varepsilon>0$, one can find a gage $\delta$ in $A$ and $\eta>0$ such that for each $\varepsilon$-regular, $\delta$-fine, $X$-tagged partition in $A\left\{\left(A_{1}, x_{1}\right), \ldots,\left(A_{p}, x_{P}\right)\right\}$ satisfying $\left|\cup_{i=1}^{p} A_{i}\right| \leq \eta$ one has

$$
\sum_{i=1}^{p}\left|F\left(A_{i}\right)\right| \leq \varepsilon .
$$

Moreover, we say that $F$ is $\mathcal{F}-A C G^{\star}(A)$ if there is a sequence $\left(X_{n}\right)_{n \in \mathbb{N}}$ of measurable subsets of $A$ such that $A=\cup_{n \in \mathbb{N}} X_{n}$ and $A$ is $\mathcal{F}-A C^{\star}\left(X_{n}\right)$.

Obviously, if $F$ is $\mathcal{F}-A C G^{\nabla}(A)$, then it is also $\mathcal{F}-A C G^{\star}(A)$. From [1], it turns out that these two concepts are equivalent in case $N=1$. What about the case $N \geq 2$ ? 
Remark 2.1 Extension to BV-integration. In the rest of this paper, the word "integrable" should be understood in the sense defined in [5], $A \subset \mathbb{R}^{N}$ will be a fixed $B V$-set and a "charge" will mean an additive function $F: B V_{A} \longrightarrow \mathbb{R}$ as defined in [5].

We may define the derivatives of a charge as in [3]. For instance,

$$
F_{\star}(x):=\inf _{\eta>0} \sup _{\delta>0} \inf \left\{\frac{F(X)}{|X|}: X \in B V_{A}, x \in c l_{e} X, d(X) \leq \delta, r(X) \geq \eta\right\} .
$$

This gives rise to a good concept of derivability for extending the Theorem 2.1 (see [3, Theorem 2.6]). However, it is not suitable for proving an analogous version of Proposition 2.1. Indeed, in the proof of this proposition, we used Vitali's covering theorem, assuming that the sets " $X$ ", taken into account in the definition of $F_{\star}$ and $F^{\star}$, are closed.

\section{References}

[1] B. Bongiorno and W. Pfeffer, A concept of absolute continuity and a Riemann type integral, Commentationes Mathematicae Universitatis Carolinae, 33(2) (1992), 189-196.

[2] J. Kurzweil and J. Jarník, Equiintegrability and controlled convergence of Perron-type integrable functions, Real Analysis Exchange, 17 (1991-92), $110-139$.

[3] W. Pfeffer, A Descriptive Definition of a Variational Integral and Apllications, Indiana University Mathematics Journal, 40(1) (1991), 259-270.

[4] W. Pfeffer, The Gauss-Green Theorem, Advances in Mathematics, 87 (1993), 93-147.

[5] W. Pfeffer, An Integral in Geometric Measure Theory, Atti Sem. Mat. Fis. Univ. Modena, XLI (1993), 59-76.

[6] W. Pfeffer, The Riemann approach to integration. Local geometric theory, Cambridge University Press, Cambridge Tracts in Mathematics, 109, Cambridge-New York-Melbourne, 1993.

[7] S. Saks, Theory of the integral, Dover Publications, Inc. Second revised ed., New York, 1964. 